\title{
Medical school weight gain understudied
}

\author{
Cite as: CMAJ 2019 October 15;191:E1145. doi: 10.1503/cmaj.1095818
}

Posted on cmajnews.com on Sept. 26, 2019

$\mathbf{T}$

he Freshman 15 is mostly a myth, but the link between stress eating and weight gain is real and understudied in medical trainees.

Students do gain weight in university, just not as much as people think, says Mallory Frayn, a doctoral student in clinical psychology at McGill University who studies relationships with food. One recent Canadian study found that female students gained an average of four pounds during freshman year, and male students gained eight pounds, largely because they consumed more alcohol and fried food.

Eating to cope with stress plays a role in the phenomenon, says Frayn. Studies link school stress to emotional eating, and emotional eating to weight gain and trouble losing weight.

And it's not just a freshman problem, Frayn notes. Medical education is potentially a perfect storm for stress eating but not much is known about the prevalence among trainees.

All the factors that contribute to emotional eating among freshman undergraduates - stress, erratic schedules, poor sleep - apply to an "exponential level" in medical school and postgraduate training, Frayn explains. "The pressure is that much higher."

Anecdotally, those posting on medical trainee forums report fluctuations in weight during medical school linked to changes in stress levels, diets and workloads.

The type of high achiever attracted to medical school tends to be more prone to emotional eating, too, says Frayn. High achievers may not have much expe-

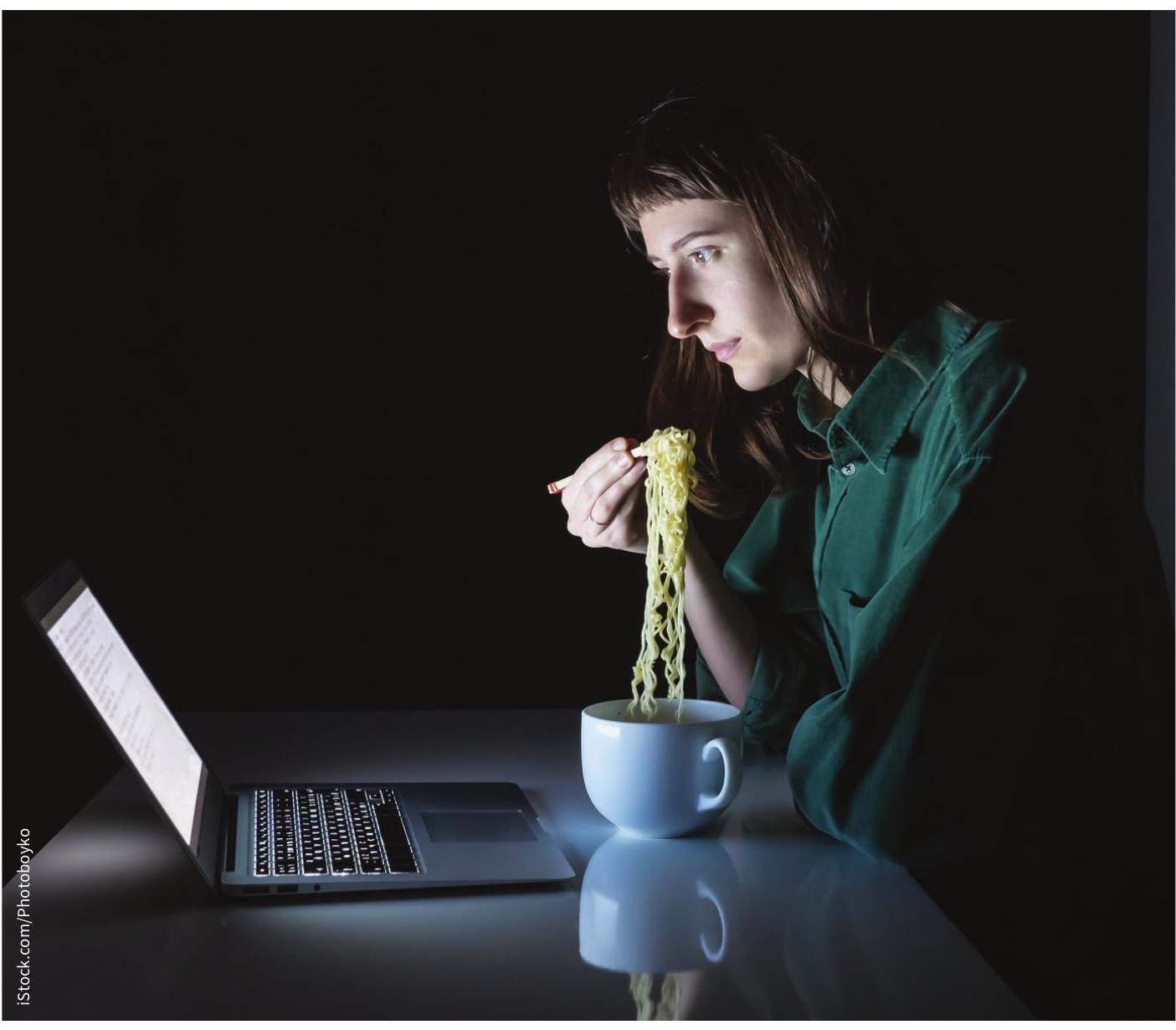

Weight gain is common among college students, but what about medical trainees?

rience with failure, so when they inevitably face setbacks in medical training, "they don't have the coping mechanisms to deal with that."

Medicine's culture of self-sacrifice may also make it harder for trainees to take time out for other activities that reduce stress, like exercise and socializing, Frayn says. "It's almost glorified, this idea that we never sleep, we work hard, we have 12to 14-hour shifts."

Comfort eating isn't a bad coping strategy in and of itself, she notes. But for medical trainees, gaining weight may be a first indicator that they need to develop more outlets for stress.

Frayn's research shows emotional eaters who maintain a normal weight often report offsetting comfort eating with exercise. "Not that exercise is a good method to facilitate weight loss, but a sedentary lifestyle is associated with weight gain," she says. Her research also suggests mindfulness techniques may be protective against weight gain from emotional eating.

Lauren Vogel, CMAJ 